\title{
Röntgenographische und massenspektrometrische Untersuchung der Alkalitrimethylsilanolate
}

Aus dem Institut für Anorganische Chemie und dem Institut für Organische Chemie der Universität Hamburg

(Eingegangen am 17. November 1969)

Die Kristallstrukturen der isotypen Trimethylsilanolate $\left(\mathrm{CH}_{3}\right)_{3} \operatorname{SiOM}(\mathrm{M}=\mathrm{K}, \mathrm{Rb}, \mathrm{Cs})$ wurden aus $P$ ulverdaten bestimmt. Die Verbindungen kristallisieren kubisch-primitiv (Raumgruppe $\mathrm{P} \overline{4} 3 \mathrm{~m})$ und bilden wie die analogen tert.-Butylate, $\left[\left(\mathrm{CH}_{3}\right)_{3} \mathrm{COM}\right]_{4}$, tetramere Struktureinheiten. Dabei besetzen die Metall- und Sauerstoffatome alternierend die Ecken eines nur wenig verzerrten Würfels, der von den vier $\left(\mathrm{CH}_{3}\right)_{3} \mathrm{Si}$-Gruppen in Richtung der verlängerten Raumdiagonalen umgeben ist. Auch im Dampfzustand sind nach massenspektrometrischen Untersuchungen tetramere Assoziate nachweisbar. Die röntgenographisch nicht näher untersuchten Trimethylsilanolate des Lithiums und Natriums treten im Massenspektrum hexamer auf.

\section{X-Ray and Mass Spectroscopic Studies of Alkali Trimethylsilanolates}

The crystal structures of the alkali trimethylsilanolates $\left(\mathrm{CH}_{3}\right)_{3} \mathrm{SiOM}(\mathrm{M}=\mathrm{K}, \mathrm{Rb}, \mathrm{Cs})$ have been determined from $X$-ray powder clata. The compounds crystallize in the cubicprimitive lattice (space group $\mathrm{P} \overline{4} 3 \mathrm{~m}$ ) and form tetrameric units, similar to the analogous tertbutoxides $\left[\left(\mathrm{CH}_{3}\right)_{3} \mathrm{COM}\right]_{4}$. In these tetramers the meta] and oxygen atoms form a slightly distorted cubic array, surrounded by four $\left(\mathrm{CH}_{3}\right)_{3} \mathrm{Si}$-groups along the directions of the extended space diagonals of the cube. In the vapor state, too, tetrameric units are existing as shown by mass spectroscopy. Lithium and sodium trimethylsilanolate have not been fully investigated by X-ray diffaction. Their mass spectra show the presence of hexamers.

Die tert.-Butylate des Kaliums, Rubidiums und Cäsiums bilden sowohl im festen als auch im gasförmigen Zustand tetramere cubanähnliche Assoziate $\left[\left(\mathrm{CH}_{3}\right)_{3} \mathrm{COM}\right]_{4}{ }^{1}$. In Weiterführung dieser Strukturuntersuchungen ließ3 sich nun zeigen, daß die Trimethylsilanolate des Kaliums, Rubidiums und Cäsiums völlig entsprechende Strukturen besitzen.

\section{Darstellung der Präparate und röntgenographische Untersuchungen}

Die Trimethylsilanolate von $\mathrm{K}, \mathrm{Rb}$ und $\mathrm{Cs}$ stellten wir nach bekannten 2) Verfahren entsprechend

$2\left(\mathrm{CH}_{3}\right)_{3} \mathrm{SiOSi}\left(\mathrm{CH}_{3}\right)_{3}+2 \mathrm{MNH}_{2} \longrightarrow 2\left(\mathrm{CH}_{3}\right)_{3} \mathrm{SiOM}+\mathrm{NH}_{3}+\left(\mathrm{CH}_{3}\right)_{3} \mathrm{SiNHSi}\left(\mathrm{CH}_{3}\right)_{3}$

1) E. Weiss, H. Alsdorf, H. Kühr und H.-F. Grützmacher, Chem. Ber. 101, 3777 (1968).

2) F.J.Hyde, O.K. Johannson, W. H. Daudt, R.F.Fleming, H. B. Laudenslager und M. P. Roche, J. Amer. chem. Soc. 75, 5615 (1953). 
aus dem Alkaliamid und Hcxamethyldisiloxan ${ }^{3)}$ in flüssigem Ammoniak dar. Sie wurden nach Abziehen der flüchtigen Bestandteile mehrfach mit Benzol/Äther gewaschen, bei $20^{\circ} / 10^{-3}$ Torr getrocknet und so in ausgezeichneter Reinheit erhalten. Die Na-Verbindung wurde in entsprechender Weise, jedoch in Äther als Lösungsmittel, dargestellt. Eine Reinigung ist hier durch Sublination $\left(140^{\circ} / 10^{-3}\right.$ Torr) möglich, desgleichen bei der Li-Verbindung4), die durch Umsetzung von metallischem Lithium mit Trimethylsilanol5) gewonnen wurde. Da die Trimethylsilanolate äußerst hydrolysenempfindlich sind, wurden dic Operationen unter getrocknetem Rcinststickstoff und unter Verwendung gründlich absolutierter Lösungsmittel ausgeführt.

Die röntgenographische Untersuchung erfolgte unter Verwendung von $\mathrm{Cu}-\mathrm{K}_{\alpha_{1}}$-Strahlung $(\lambda=1.5405 \AA)$ mit Hilfe eines Zählrohrgoniometers 1$)$.

$\mathrm{K}$-, $\mathbf{R b}$ - und $\mathrm{Cs}$-Trimethylsilanolat kristallisieren kubisch mit den in Tab. 1 aufgeführten Zellkonstanten und röntgenographischen Dichten.

Tab. 1. Elementarzellen und Dichten der Alkalitrimethylsilanolate

\begin{tabular}{|c|c|c|c|}
\hline & K & $\mathrm{Rb}$ & $\mathrm{Cs}$ \\
\hline$a(\AA)$ & $8.844+0.001$ & $9.019 \pm 0.001$ & $9.295 \pm 0.002$ \\
\hline$V\left(\AA^{3}\right)$ & 691.5 & \pm 0.3 & +0.5 \\
\hline$D\left(\mathrm{~g} \cdot \mathrm{cm}^{-3}\right)$ & $1.229 \div 0.001$ & 1.5750 .001 & $1.836 \pm 0.002$ \\
\hline
\end{tabular}

Die Elementarzelle (Raumgruppe $\mathbf{P} \overline{4} 3 m$ ) enthält eine tetramere Einheit $\left[\left(\mathrm{CH}_{3}\right)_{3} \mathrm{SiOM}\right]_{4}$, deren Atomlagen, wie bereits früher beschrieben 1', ermittelt wurden. Es seien daher hier nur die Ergebnisse mitgeteilt.

Für die Besetzung der Punktlagen M,O und Si auf $(x, x, x) ;(x, \bar{x}, \bar{x}) ;(\bar{x}, x, \bar{x}) ;(\bar{x}, \bar{x}, x)$ und $\mathrm{CH}_{3}$ (Schwerpunkte) auf $(x, x, z) ;(z, x, x) ;(x, z, x) ;(\bar{x}, x, \bar{z}) ;(\bar{z}, x, \bar{x}) ;(\bar{x}, z, \bar{x}) ;(x, \bar{x}, \bar{z})$; $(z, \bar{x}, \bar{x}) ;(x, \bar{z}, \bar{x}) ;(\bar{x}, \bar{x}, z) ;(\bar{z}, \bar{x}, x) ;(\bar{x}, \bar{z}, x)$ resultierten die in Tab. 2 angegebenen Lagenparameter.

Tab. 2. Atomkoordinaten der Alkalitrimethylsilanolate

\begin{tabular}{lcll}
\hline & $\mathrm{K}$ & \multicolumn{1}{c}{$\mathrm{Rb}$} & \multicolumn{1}{c}{$\mathrm{Cs}$} \\
\hline$x_{\mathrm{M}}$ & $0.350 \pm 0.002$ & $0.344 \pm 0.002$ & $0.340 \pm 0.002$ \\
$x_{\mathrm{O}}$ & $0.644 \pm 0.004$ & $0.647 \mathrm{a})$ & $0.654 \mathrm{~b})$ \\
$x_{\mathrm{Si}}$ & $0.749 \pm 0.004$ & $0.750 \pm 0.004$ & $0.755 \mathrm{~b})$ \\
$x_{\mathrm{CH}}$ & $0.712 \pm 0.005$ & $0.710 \pm 0.005$ & $0.721 \mathrm{~b})$ \\
$z_{\mathrm{CH} 3}$ & $0.945 \pm 0.005$ & $0.945 \pm 0.005$ & $0.939 \mathrm{~b})$
\end{tabular}

a) Durch normalen Si-O-Abstand ( $1.61 \AA$ ) festgelegt.

b) Durch normale Si-O- und Si-C- $(1,83 \AA)$ Abstände festgelegt.

Genaue Atomlagenbestimmungen (mit Ausnahme der nicht lokalisierbaren $\mathrm{H}$-Atome) waren bei der $\mathrm{K}$ - und Rb-Verbindung möglich. Bei der Cs-Verbindung wurden wegen der geringeren Qualität der Beugungsdiagramme und wegen des dominierenden Streuanteils der Cs-Atome nur deren Lagen experimentell bestimmt und die übrigen Atomkoordinaten aus bekannten Bindungsabständen festgelegt.

3) R. O. Sauer, J. Amer. chem. Soc. 66, 1707 (1944).

4) W. S. Tatlock und E. G. Rochow, J. org. Chemistry 17, 1555 (1952).

5) L. H. Sommer, E. W. Pietrusza und F. C. Whitmore, J. Amer. chem. Soc. 68, 2282 (1946). 
Tab. 3. Vergleich von berechneten und beobachteten Netzebenenabständen und Intensitäten

\begin{tabular}{|c|c|c|c|c|}
\hline 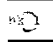 & 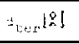 & 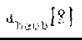 & $\perp_{1, e}=$ & Feobs \\
\hline 01 & $3.341 ?$ & 8,80 & 90,2 & 210.6 \\
\hline $\begin{array}{lll}01 & 1 \\
11 & 1\end{array}$ & 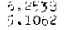 & $5: 26$ & $7 . * 4$ & 54 \\
\hline $\begin{array}{ll}101 \\
012 \\
0\end{array}$ & $\begin{array}{r}+4220 \\
3.4553\end{array}$ & 1.14 & 36,31 & 94 \\
\hline 112 & $\begin{array}{l}3.0703 \\
3.0006\end{array}$ & 3.611 & $\frac{1}{10.39}$ & 25 \\
\hline$\frac{2}{5}$ & 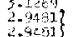 & $\begin{array}{l}3.214 \\
2.547\end{array}$ & $99.05,6 \%$ & 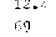 \\
\hline 列 & $\begin{array}{l}2.40912 \\
2.7906\end{array}$ & a.79\% & $\$ .17$ & $\therefore$; \\
\hline 41 & $\begin{array}{l}2.6656 \\
2.55 \%)\end{array}$ & $\begin{array}{l}2.667 \% \\
2.5544\end{array}$ & 20.09 & $=0$ \\
\hline $\begin{array}{l}2,5 \\
123\end{array}$ & $\begin{array}{l}2.250 \\
2.420 \\
2.3655\end{array}$ & $\begin{array}{l}n+50 \% \\
3.31 \%\end{array}$ & $\begin{array}{l}0.46 \\
5.06 \\
0.54\end{array}$ & $\begin{array}{l}2.0 \\
3.3 \\
i .3\end{array}$ \\
\hline of & $\begin{array}{l}2.2113 \\
2=1150 \\
2=140\end{array}$ & & $\begin{array}{l}0.05 \\
0.904\end{array}$ & $\frac{1}{6} \cdot 3$ \\
\hline $\begin{array}{l}223 \\
1113\end{array}$ & $\begin{array}{r}1450\} \\
2.1450 \\
0.0206\end{array}$ & 201454 & $0.0\}$ & 3.5 \\
\hline 03 & 2.5BA4 is $\}$ & $\begin{array}{l}2.03 n 5 \\
2.02 y_{2}\end{array}$ & 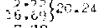 & 16.7 \\
\hline & $\begin{array}{l}2.0290 \\
1.34\end{array}$ & $\begin{array}{l}2.0252 \\
1.0 \% \%\end{array}$ & 7.10 & 12.8 \\
\hline 33 & $\begin{array}{r}060 \\
305\end{array}$ & $\begin{array}{l}1.9760 \\
1.901\end{array}$ & $\begin{array}{l}2.44 \\
4.065\end{array}$ & 3.3 \\
\hline 325 & $\begin{array}{r}1.055 \\
\therefore .4055 \\
1.56\end{array}$ & $\begin{array}{l}\text { 1. } 50502 \\
1.0450\end{array}$ & $\frac{2.30}{3.23}$ & 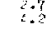 \\
\hline $\begin{array}{l}005 \\
09 \\
0\end{array}$ & $\left.\begin{array}{l}1.7584 \\
1.76096\end{array}\right\}$ & 1.9585 & 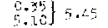 & 6.6 \\
\hline 01 & $\begin{array}{l}1.73+5 \\
1.73 \leq 5\end{array}$ & $1.734:$ & $\begin{array}{l}0.22 \\
5.47\end{array} \quad 6.70$ & 7.2 \\
\hline$\frac{17}{35}$ & $\begin{array}{l}7.90214 \\
1.9021\end{array}$ & 1.7082 & $\left.\begin{array}{r}12.17 \\
3.53\end{array}\right] 15.70$ & 15.7 \\
\hline 025 & $\begin{array}{l}1.6429 \\
1.6425\end{array}$ & 2.500 & $\{.72\} 0.76$ & 1.5 \\
\hline $\begin{array}{ll}125 \\
0<4\end{array}$ & $\begin{array}{l}1.6117 \\
1.5655\end{array}$ & $\begin{array}{l}2.6142 \\
1.56,30\end{array}$ & $\begin{array}{l}1<2 \\
3,27\end{array}$ & 3.2 \\
\hline $\begin{array}{ll}14 & 4 \\
22 & 5\end{array}$ & $\begin{array}{l}1.5396\} \\
1.5396\end{array}$ & 1.5391 & $1.01\} 2.28$ & 2.1 \\
\hline $\begin{array}{l}05 \\
334 \\
334\end{array}$ & $\begin{array}{l}1.5160 \\
2.516\end{array}$ & $1.51=5$ & $0.15\}=.72$ & 3.1 \\
\hline 155 & 2.1945 & 1.4919 & 3.69 & 3.1 \\
\hline$\infty 5$ & $\begin{array}{l}1.1790 \\
2 .+440\end{array}$ & $2.4 \times 0$ & $0.01\} \times .03$ & $\therefore$ \\
\hline $\begin{array}{lll}4 & 3 \\
01 & 6\end{array}$ & 1.4540 & 1.5435 & $0.90^{\circ}$ & 0.8 \\
\hline$\frac{12}{2}=6$ & $\begin{array}{l}1.4517 \\
1.25,7\}\end{array}$ & 1.1347 & $\left.\begin{array}{l}0.14 \\
0.02\end{array}\right\} 0.54$ & 0.6 \\
\hline $02 \frac{11}{94}$ & $\begin{array}{l}1.3951 \\
\frac{1}{2.3812}\end{array}$ & $1.5 \mathrm{5} \mathrm{k} 2$ & $\begin{array}{l}0.74 \\
0.73\end{array}$ & 3.2 \\
\hline 32 & $\left.\begin{array}{l}1.3812 \\
1.3812\end{array}\right\}$ & 1.3012 & $0.25\} 2.15$ & 1.9 \\
\hline $\begin{array}{ll}14 \\
33 \\
5\end{array}$ & $\begin{array}{l}3547 \\
1.3487\end{array}$ & $\begin{array}{l}3.304 \theta \\
2.3484\end{array}$ & 1.35 & 8.6 \\
\hline 325 & $\begin{array}{l}1.3481 \\
1.3555\end{array}$ & 3.3935 & $\begin{array}{l}3.24 \\
0.92\end{array}$ & 1.3 \\
\hline $\begin{array}{ll}0 x \\
21 & 6 \\
24\end{array}$ & $\left.\begin{array}{l}1.3184 \\
1 . x \geq 84\end{array}\right\}$ & 1.5181 & $\begin{array}{l}1.23 \\
0.72\} 1.85\end{array}$ & 1.8 \\
\hline $\begin{array}{lll}23 & 6 \\
44 & 4\end{array}$ & $\begin{array}{l}1.3040 \\
1.2765\end{array}$ & 1.3059 & $\begin{array}{l}1.24 \\
0.06\end{array}$ & $c^{2.3}$ \\
\hline $\begin{array}{ll}007 \\
23 & 6\end{array}$ & $\begin{array}{l}\{.2635 \\
1.2635\end{array}$ & 1. 2631 & $\left.\begin{array}{l}0.29 \\
0.98\end{array}\right\} 0.5 .7$ & 1.1 \\
\hline $\begin{array}{l}107 \\
055 \\
345\end{array}$ & $\left.\begin{array}{l}1.2506 \\
1.2508 \\
1.250 \mathrm{~g}\end{array}\right\}$ & 1.2505 & $\left.\begin{array}{l}1.05 \\
0.04 \\
0.05\end{array}\right\} 1.14$ & 1.0 \\
\hline$\frac{117}{15 \div 3}$ & $\begin{array}{r}1.23 \mathrm{E} 4 \\
1.2704\end{array}$ & 2.2355 & $1.101,1.31$ & $\ldots 1$ \\
\hline 04 & 1.2265 & 1.2255 & 3.10 & 2.7 \\
\hline $\begin{array}{l}0 \% \\
146 \\
6\end{array}$ & $\begin{array}{l}1.2245 \\
1.2149\end{array} \mid$ & 1.6149 & $\left.\begin{array}{c}c .212 \\
c .69\end{array}\right\} 0.00$ & 0.9 \\
\hline $\begin{array}{l}12 \\
25 \\
25 \\
x=5 \\
5\end{array}$ & $\begin{array}{l}1.203 \\
1+2055 \\
\end{array}$ & $\therefore 20 \%$ & $\begin{array}{r}0.43 \\
0.02 \\
0.000\end{array}$ & 1.3 \\
\hline 246 & 1.1019 & $1.2 \leqslant 183$ & 2.07 & 2.5 \\
\hline & $\left\{\begin{array}{l}7.174 \\
1.2724\end{array}\right\}$ & 1.1719 & $\left.\begin{array}{l}0.25 \\
0.25\end{array}\right\} 0.51$ & 0.5 \\
\hline $\begin{array}{l}53 \\
137\end{array}$ & $\begin{array}{l}1.2615 \\
i .1512\end{array}$ & 1.1625 & $\begin{array}{l}0.65 \\
1.071\end{array}$ & 0.9 \\
\hline $\begin{array}{ll}35 & 5 \\
05 & 5\end{array}$ & $\begin{array}{l}3.1514\} \\
1.1324\end{array}$ & $1.1: 2.28$ & $\begin{array}{l}6.80) \\
0.02 \\
0.02\end{array}$ & 3.5 \\
\hline 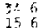 & $\begin{array}{l}1.1324\} \\
1,1 ; 5=1\end{array}$ & 1.1324 & $1.04\} 1.07$ & 0.9 \\
\hline $\begin{array}{l}257 \\
20 \\
00\end{array}$ & $\begin{array}{l}1.1233 \\
3.1055\end{array}$ & 1.1235 & $\begin{array}{l}0.24\} \\
0.30 \\
0.13\end{array}$ & 0.3 \\
\hline $\begin{array}{l}04 \% \\
25 \% \\
62 \%\end{array}$ & $\left.\begin{array}{c}0978 \\
30170 \\
30070\end{array}\right]$ & $1.01 \%$ & $3.73\} 0.95$ & c.6 \\
\hline $\begin{array}{ll}14 & 7 \\
11 & 8 \\
45 & 5\end{array}$ & $\left.\begin{array}{r}1.0357 \\
3.0237\end{array}\right\}$ & $\therefore, 0000$ & $\begin{array}{ll}0.27 \\
0.22 & 0.62\end{array}$ & 0.4 \\
\hline 35 & $\begin{array}{l}1.01267,2 \\
3.0305,\end{array}$ & $1.0: 0$ & 8.05 & $\therefore$ \\
\hline$\therefore$ & $0,0<3\}$ & 1.0729 & (6) & $8 \%$ \\
\hline $\begin{array}{ll}24 & 2 \\
12 & 8\end{array}$ & $\left.\begin{array}{r}2.064 \% \\
1.0547\end{array}\right\}$ & $\therefore .054 ?$ & $\begin{array}{c}0.05 \\
0.25\end{array} 0.30$ & 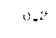 \\
\hline
\end{tabular}

\begin{tabular}{|c|c|c|c|c|}
\hline$x_{-1}^{-1}$ & $b_{b=2}\left(a_{1}\right.$ & $a_{\text {besh }}|y|$ & $I_{b=x}$ & $I_{\text {beol }}$ \\
\hline 53 & $\begin{array}{r}0013 \\
0.0423\}\end{array}$ & $1.0: 83$ & $\begin{array}{c}0.01 \\
0.011\end{array}$ & 0.9 \\
\hline 225 & $\begin{array}{r}2.025\} \\
3.0341\}\end{array}$ & 1.0552 & $\begin{array}{l}0.411 \\
0.7 y\} \\
0.21\end{array}$ & \\
\hline $\begin{array}{l}0,5 \\
0.5 \\
0.3 \\
3 \\
3\end{array}$ & $\left.\begin{array}{r}1.031\} \\
3.02031\end{array}\right\}$ & & & 0.3 \\
\hline 31 & 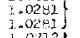 & $\therefore .0253$ & {$\left[\begin{array}{c}0.89 \\
0.09\end{array}\right] 0.40$} & 0.5 \\
\hline $\begin{array}{c}57 \\
55 \\
5\end{array}$ & $\left.\begin{array}{l}1.0225 \\
1.0212\end{array}\right\}$ & 1.0213 & $\left.\begin{array}{l}0.23 \\
0.35\end{array}\right\} 0.50$ & 0.5 \\
\hline $8 \pi$ & 1.025 & 2.0145 & 0.55 & 0.5 \\
\hline 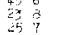 & $\{0009$ & 1.0079 & 0.0010 .09 & 0.2 \\
\hline 803 & 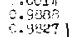 & & $\begin{array}{l}0.03 \\
0.03 \\
0.00\end{array}$ & \\
\hline 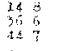 & $\left.\begin{array}{l}6.927 \\
0.4027 \\
0.4 \geq 2 ?\}\end{array}\right\}$ & 0.3825 & $\left.\begin{array}{c}0.37 \\
0.25 \\
0.25\end{array}\right\}^{0.90}$ & 0.2 \\
\hline $\begin{array}{ll}019 \\
y=4\end{array}$ & $\left.\begin{array}{r}0.4767 \\
8.4767\end{array}\right\}$ & 0.9757 & $\left.\begin{array}{c}0.08 \\
0.03\end{array}\right\} 0.13$ & 0.1 \\
\hline $\begin{array}{lll}i 1 & 9 \\
3 & 9\end{array}$ & $\begin{array}{r}0.9705 \\
8.9709\end{array}$ & 0.9707 & $\left.\begin{array}{l}0.08 \\
0.4\end{array}\right\} 0.55$ & 0.7 \\
\hline $\begin{array}{l}24 \\
04 \\
02\end{array}$ & $\left.\begin{array}{r}.0650 \\
0.9595 \\
c .959 y 3\end{array}\right\}$ & 0.9618 & $\begin{array}{l}0.37 \\
0.40 \\
0.40\end{array}$ & 0.4 \\
\hline $\begin{array}{rl}26 & 0 \\
26 & 6 \\
32 & 6 \\
32 & 9\end{array}$ & 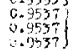 & $x .9 \% 3 ?$ & $\left.\begin{array}{r}0.24 \\
0.10 \\
8.12\end{array}\right\} .45$ & 0.3 \\
\hline $\begin{array}{l}456 \\
35.8\end{array}$ & $\begin{array}{l}0.9423 \\
0.9375\end{array}$ & & 5.02 & 0 \\
\hline 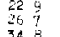 & $\left.\begin{array}{r}0.975 \\
8.9375\end{array}\right\}$ & $0.13 \% 6$ & $\left.\begin{array}{l}0.05 \\
0.1\end{array}\right\} 0.50$ & 0.3 \\
\hline $\begin{array}{rl}348 \\
059 \\
03 & 9\end{array}$ & $\begin{array}{l}0.9375] \\
0.93231\end{array}$ & & $\begin{array}{l}0.12 \\
0.03\end{array}$ & \\
\hline $\begin{array}{l}158 \\
457\end{array}$ & $\begin{array}{l}0.01523 \\
0.9323\end{array}$ & 0.3324 & {$\left[\begin{array}{c}0.08 \\
0.04\end{array}\right\}^{0.12}$} & 0.5 \\
\hline $\begin{array}{l}73 \\
25 \\
25\end{array}$ & $\begin{array}{l}0.9871 \\
0.4717\end{array}$ & $\begin{array}{l}0.9271 \\
0.971\end{array}$ & $\begin{array}{l}6.60 \\
0.24\end{array}$ & 0.4 \\
\hline 309 & $\left.\begin{array}{r}0.9122 \\
0.9122\end{array}\right\}$ & 0.9123 & $\left.\begin{array}{c}0.04 \\
0.55\end{array}\right\} 0.58$ & 0.4 \\
\hline $\begin{array}{l}11 \\
56 \\
50 \\
02\end{array}$ & $\left.\begin{array}{r}0.9027 \\
0.8060 \\
0.8050\end{array}\right\}$ & 0.5027 & $\left.\begin{array}{l}0.41 \\
0.02 \\
0.32\end{array}\right\} 0.22$ & $\begin{array}{l}0.2 \\
0\end{array}$ \\
\hline $\begin{array}{ll}07 & 7 \\
3 & 8 \\
14 & 8 \\
9 & 9\end{array}$ & $\left.\begin{array}{l}0.3934 \\
0.3934 \\
0.3934\end{array}\right\}$ & 0.8934 & $\begin{array}{l}0.26 \\
0.09 \\
c .15 j\end{array} \quad 0.30$ & 6.4 \\
\hline $37 \frac{7}{3}$ & 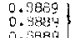 & $0.8 \% \times 19$ & \begin{tabular}{l|l|l}
0.19 & 0.74
\end{tabular} & 0.7 \\
\hline co io & $\left.\begin{array}{r}0.0 .44 \\
0.044\end{array}\right]$ & 0.2943 & $\left.\begin{array}{r|c}0.25 \\
0.23\end{array}\right] .28$ & 0.5 \\
\hline 10 & $\begin{array}{l}0.8000 \\
c .8800\end{array}$ & 0.5000 & $\begin{array}{r}0.25 \\
0.09\end{array}$ & \\
\hline $45 \%$ & $\left.\begin{array}{l}0.0300 \\
0.3800\end{array}\right]$ & & $\begin{array}{l}0.09 \\
0.30\end{array}$ & \\
\hline$\frac{11}{20} 7$ & $\begin{array}{l}0.891 \\
0.07,7]\end{array}$ & & $\begin{array}{l}0.09 \\
0.00\end{array} 0.10$ & 0 \\
\hline$\frac{10}{8}$ & $\left.\begin{array}{l}0.8673 \\
0.8673\end{array}\right]$ & $0.96 \% 1$ & $\begin{array}{l}0.25 \\
0.9608\end{array}$ & 0.6 \\
\hline & $\left.\begin{array}{l}0.1851 \\
0.063: \ldots\end{array}\right]$ & & $\left.\begin{array}{r}8.10 \\
0.02\end{array}\right\} 0.22$ & c \\
\hline & 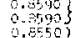 & & $\left.\begin{array}{l}0.65 \\
0.25\end{array}\right\} 0.30$ & 0 \\
\hline 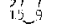 & c.est & $(6,8) 44$ & $\begin{array}{l}0.64 \\
0.67\end{array} 1.07$ & 1.2 \\
\hline
\end{tabular}

Den Atomkoordinaten der Tab. 2 entsprechen die in Tab. 3 aufgeführten Streuintensitäten. Die Übereinstimmung zwischen beobachteten und berechneten Intensitäten (und Netzebenenabständen) ist sehr zufriedenstellend. Der Zuverlässigkeitsindex $R=100 \Sigma\left|I_{\text {beob }}-I_{\text {ber }}\right| \Sigma I_{\text {beab }}$ ergab sich zu $7.9 \%$ (K), $7.2 \%$ (Rb), $13.5 \%$ (Cs). Unter Ansatz isotroper, durchschnittlicher Temperaturfaktoren betrugen deren Koeffizienten $B=3.1 \AA^{2}(\mathrm{~K}), 4.5 \AA^{2}(\mathrm{Rb})$ und $5.1 \AA^{2}(\mathrm{Cs})$. 
Tab. 3 (Fortsetzung)

\begin{tabular}{|c|c|c|c|c|}
\hline nes & $\mathrm{d}_{\text {ter }}\{\mathrm{l}\}$ & $a_{\text {teoio }}[\mathrm{d}]$ & ${ }^{I_{\text {yex: }}}$ & $I_{z e c b}$ \\
\hline $\begin{array}{ll}00 & 1 \\
01 & 1 \\
011 & 1 \\
11 & 1 \\
00 & 2 \\
01 & 2 \\
11 & 2 \\
02 & 2 \\
00 & 3 \\
12 & 2 \\
01 & 3\end{array}$ & $\begin{array}{l}9.0195 \\
6.3776 \\
5.2773 \\
4.5047 \\
4.0536 \\
3.6871 \\
3.1348 \\
3.0054 \\
3.0064 \\
2.5512\end{array}$ & $\begin{array}{l}9.01 \\
0.37 \\
6.202 \\
6.505 \\
4.051 \\
3.012 \\
3.189 \\
5.004 \\
2.851\end{array}$ & 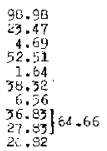 & $\begin{array}{l}100.0 \\
22 \\
4.4 \\
3.1 \\
1.6 \\
38 \\
3.3 \\
67 \\
21\end{array}$ \\
\hline 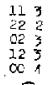 & $\begin{array}{l}2.7194 \\
2.6057 \\
2.5015 \\
2.4705 \\
2.2540\end{array}$ & $\begin{array}{l}2.7984 \\
2.6026 \\
2.5013 \\
2.4105 \\
2.2550\end{array}$ & $\begin{array}{r}11.133 \\
6.58 \\
8.07 \\
1.50 \\
0.45\end{array}$ & $\begin{array}{r}12 \\
3.8 \\
0.2 \\
2.4 \\
1.5\end{array}$ \\
\hline $\begin{array}{ll}22 & 3 \\
01 & 4 \\
03 & 3 \\
11 & 4 \\
13 & 3 \\
02 & 1 \\
12 & 4 \\
23 & 3 \\
22 & 4\end{array}$ & $\begin{array}{l}2.1815 \\
2.1975\} \\
2.1259 \\
2.1259 \\
2.0692 \\
2.0168 \\
1.9682 \\
1.9229 \\
1.8 .15\end{array}$ & $\begin{array}{l}2.1877 \\
2.1262 \\
2.0692 \\
1.9685 \\
2.9271 \\
1.9414\end{array}$ & 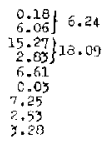 & $\begin{array}{r}5.8 \\
20.6 \\
5.9 \\
0.9 \\
8.9 \\
2.2 \\
2.3\end{array}$ \\
\hline $\begin{array}{ll}00 & 5 \\
03 & 4 \\
13 & 4 \\
21 & 5 \\
02 & 5 \\
23 & 4 \\
12 & 5 \\
04 & 4 \\
14 & 4 \\
28 & 5\end{array}$ & 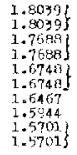 & $\begin{array}{l}2.0057 \\
1.7691 \\
1.5750 \\
1.5459 \\
1.5913 \\
1.5700\end{array}$ & 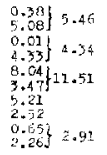 & $\begin{array}{r}5.5 \\
4.0 \\
1.8 \\
5.2 \\
2.2 \\
2.7\end{array}$ \\
\hline $\begin{array}{ll}05 & 5 \\
35 & 5 \\
13 & 5 \\
00 & 5 \\
21 & 4 \\
01 & 6 \\
11 & 5 \\
23 & 5 \\
02 & 5\end{array}$ & $\begin{array}{l}1.5469 \\
1.5468\} \\
1.5255 \\
1.5032 \\
1.5032 \\
1.428 \\
1.4531 \\
1.451 \\
1.4261\end{array}$ & $\begin{array}{l}1.5468 \\
1.5247 \\
1.5032 \\
1.4826 \\
2.4631\end{array}$ & 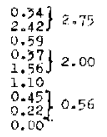 & $\begin{array}{l}2.4 \\
0.7 \\
2.3 \\
1.2 \\
0.3 \\
0\end{array}$ \\
\hline $\begin{array}{ll}04 & 5 \\
12 & 6 \\
34 & 4 \\
14 & 5 \\
35 & 5 \\
22 & 5 \\
05 & 6 \\
24 & 5 \\
15 & 6 \\
44 & 4\end{array}$ & $\begin{array}{l}1.4006 \\
3.4006 \\
1.4006\} \\
1.3417 \\
1.3754 \\
1.3597 \\
1.3445 \\
1.3445 \\
1.3298 \\
1.3018\end{array}$ & $\begin{array}{l}1.4004 \\
1.3916 \\
1.3755 \\
-.3596 \\
1.5426 \\
1.3297\end{array}$ & $\begin{array}{l}0.01] \\
0.32 \\
1.04 \\
1.01 \\
0.43 \\
0.16 \\
1.701 .35 \\
1.40 .12 \\
1.20 \\
0.01\end{array}$ & $\begin{array}{l}1.4 \\
1.3 \\
0.4 \\
0.3 \\
3.3 \\
1.6 \\
0\end{array}$ \\
\hline $\begin{array}{ll}00 & 7 \\
23 & 6 \\
01 & 7 \\
05 & 5 \\
34 & 5 \\
1 & 5 \\
15 & 7 \\
04 & 5 \\
02 & 5 \\
02 & 7 \\
24 & 5\end{array}$ & $\left.\begin{array}{l}1.2885 \\
1.2985 \\
1.2755 \\
1.2755 \\
1.2755 \\
1.2650 \\
1.2630 \\
1.2508 \\
1.2383 \\
1.2350\end{array}\right\}$ & $\begin{array}{l}1.2884 \\
1.2754 \\
1.2629 \\
1.2507 \\
1.2310\end{array}$ & 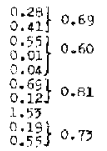 & $\begin{array}{l}1.1 \\
1.7 \\
0.9\end{array}$ \\
\hline $\begin{array}{l}127 \\
255 \\
3>6 \\
245 \\
227 \\
245 \\
097 \\
137 \\
359\end{array}$ & $\left.\begin{array}{l}1.2274 \\
1.2274 \\
1.2271 \\
1.2053 \\
1.194 E \\
1.1946 \\
1.1945 \\
1.1742 \\
1.1742\end{array}\right\}$ & $\begin{array}{l}1.2273 \\
1.2054 \\
1.1917 \\
1.1845 \\
1.1742\end{array}$ & 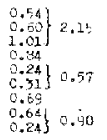 & $\begin{array}{l}2.3 \\
1.3 \\
0.5 \\
1.3 \\
1.1\end{array}$ \\
\hline $\begin{array}{ll}05 & 6 \\
34 & 5 \\
23 & 7 \\
15 & 6 \\
00 & 8 \\
01 & 5 \\
04 & 7 \\
25 & 6 \\
11 & 8 \\
14 & 7 \\
45 & 5\end{array}$ & $\left.\begin{array}{l}1.1544 \\
1.1548 \\
1.1455 \\
1.1455 \\
1.1274 \\
1.1187 \\
1.1189 \\
1.1197 \\
1.1102 \\
1.1102 \\
1.1102\end{array}\right\}$ & $\begin{array}{l}1.1548 \\
1.12566 \\
1.11088 \\
1.1103\end{array}$ & 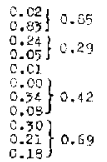 & $\begin{array}{l}1.1 \\
0.5 \\
0 \\
0.5\end{array}$ \\
\hline
\end{tabular}

\begin{tabular}{|c|c|c|c|c|c|}
\hline $\mathrm{kn}_{\mathrm{k}}$ & $a_{b e r}[R]$ & $d_{\text {bect }}[\AA]$ & $I_{\text {ber }}$ & & $I_{\text {beok }}$ \\
\hline \multirow{5}{*}{$\begin{array}{ll}3 & 7 \\
02 & 8 \\
44 & 6 \\
12 & 8 \\
24 & ? \\
33 & 6 \\
0 & 6 \\
22 & 8 \\
05 & 8 \\
16 & 6\end{array}$} & \multirow{5}{*}{$\left.\begin{array}{l}1.1029 \\
1.0038 \\
1.0938 \\
1.0058 \\
1.0058 \\
1.0780 \\
1.0629 \\
1.0529 \\
1.0555 \\
1.0556\end{array}\right\}$} & 1.2020 & $\begin{array}{l}0.51 \\
0.04\end{array}$ & 0,10 & 0.7 \\
\hline & & $\therefore .0859$ & $\begin{array}{l}0.061 \\
0.45\end{array}$ & 0.60 & 1.0 \\
\hline & & & $\begin{array}{l}0.255 \\
0.02\end{array}$ & & 0 \\
\hline & & 1.0650 & $\begin{array}{l}0.35 \\
0.28\end{array}$ & 0.64 & 1.0 \\
\hline & & 2.0558 & $\begin{array}{l}0.00 \\
0.13\end{array}$ & 0.14 & 0.2 \\
\hline \multirow{5}{*}{$\begin{array}{ll}03 & 7 \\
13 & 8 \\
31 & 7 \\
15 & 7 \\
55 & 5 \\
26 & 6 \\
23 & 8 \\
45 & 6 \\
25 & 7 \\
04 & 8\end{array}$} & \multirow{5}{*}{$\left.\begin{array}{l}1.0485 \\
1.0455 \\
3.0285 \\
1.0425 \\
1.0415 \\
1.0346 \\
1.0278 \\
1.0278 \\
1.0212 \\
1.0024\end{array}\right\}$} & 1.0485 & $\left.\begin{array}{l}0.11 \\
0.02 \\
0.31\end{array}\right\}$ & 0.44. & 0.5 \\
\hline & & 1.0413 & 0.09 & 0.23 & 0.3 \\
\hline & & 1.0345 & 0.20 & & 0.2 \\
\hline & & & $\left.\begin{array}{l}0.03 \\
0.03\end{array}\right\}$ & 0.06 & 0 \\
\hline & & 1.0212 & $\begin{array}{l}0.19 \\
0.01\end{array}$ & & 0.2 \\
\hline \multirow{9}{*}{$\begin{array}{ll}00 & 9 \\
14 & 8 \\
36 & 5 \\
44 & 7 \\
07 & 9 \\
33 & 8 \\
11 & 9 \\
35 & 7 \\
24 & 8 \\
02 & 5 \\
06 & 7 \\
12 & 9 \\
16 & 7 \\
55 & 5 \\
46 & 6 \\
05 & 8 \\
22 & 9 \\
26 & 7 \\
34 & 8\end{array}$} & & & \\
\hline & $\begin{array}{l}1.0021 \\
1.0021\end{array}$ & 1.0021 & $\begin{array}{l}0.27 \\
0.10\end{array}$ & 0.69 & 0.9 \\
\hline & $\left.\begin{array}{l}0.9360 \\
0.9660\end{array}\right\}$ & & $\left.\begin{array}{l}0.08 \\
0.00\end{array}\right]$ & 0.04 & 0 \\
\hline & $\left.\begin{array}{l}0.9900 \\
0.9900\end{array}\right\}$ & 0.5900 & $\left.\begin{array}{l}0.09 \\
0.11\end{array}\right]$ & 0.24 & 0.3 \\
\hline & 0.9841 & 0.9840 & 0.26 & & 0.4 \\
\hline & $\begin{array}{l}0.9783 \\
0.9783\end{array}$ & 0.9781 & $\left.\begin{array}{l}0.01 \\
0.23\end{array}\right\}$ & 0.25 & 0.4 \\
\hline & $\begin{array}{l}C .9726 \\
0.9726\end{array}$ & 0.9726 & $\left.\begin{array}{l}0.09 \\
0.16\end{array}\right\}$ & 0.28 & 0.5 \\
\hline & $\begin{array}{l}0.9720 \\
0.9615 \\
0.0560\end{array}$ & & $\begin{array}{l}0.031 \\
0.03 \\
0.001\end{array}$ & & 0 \\
\hline & $\begin{array}{l}0.9560 \\
0.9560 \\
0.9560\end{array}$ & 0.9561 & $\left.\begin{array}{l}0.04 \\
0.08 \\
0.01\end{array}\right\}$ & 0.15 & 0.2 \\
\hline \multirow{5}{*}{$\begin{array}{ll}03 & 9 \\
15 & 6 \\
45 & 7 \\
13 & 3 \\
25 & 8 \\
23 & 9 \\
36 & 7 \\
44 & 8 \\
04 & 9 \\
56 & 6\end{array}$} & \multirow{5}{*}{$\left.\begin{array}{l}0.9507 \\
0.9507 \\
0.9507 \\
0.9455 \\
0.9353 \\
0.9303 \\
0.9303 \\
0.9205 \\
0.9258 \\
0.0158\end{array}\right\}$} & 0.9208 & $\begin{array}{l}0.22 \\
0.19\end{array}$ & 0.40 & 0.9 \\
\hline & & $\begin{array}{l}0.9455 \\
0.93552\end{array}$ & $\begin{array}{l}0.31 \\
0.57\end{array}$ & & $\begin{array}{l}0.3 \\
0.5\end{array}$ \\
\hline & & 0.9902 & 0.041 & 0.37 & 0.5 \\
\hline & & & $\begin{array}{l}0.10 \\
0.11\end{array}$ & & \\
\hline & & & $0.01\}$ & 0.12 & 0 \\
\hline \multirow{4}{*}{$\begin{array}{ll}07 & 7 \\
14 & 9 \\
35 & 8 \\
17 & 7 \\
33 & 9 \\
55 & 7 \\
00 & 10 \\
06 & 9\end{array}$} & \multirow{4}{*}{$\left.\begin{array}{l}0.9111 \\
0.9111 \\
0.9111 \\
0.9055 \\
0.9055 \\
0.9065 \\
0.9019 \\
0.9019\end{array}\right\}$} & 0.91112 & $\begin{array}{l}0.06 \\
0.10\end{array}$ & 0.27 & 0.2 \\
\hline & & & 0.02 & & \\
\hline & & 0.9065 & 0.14 & 0.30 & 0.6 \\
\hline & & 0.4019 & $\left.\begin{array}{l}0.19 \\
0.01\end{array}\right\}$ & 0.20 & 0.2 \\
\hline \multirow{5}{*}{$\begin{array}{ll}01 & 10 \\
16 & 8 \\
24 & 9 \\
46 & 7 \\
1.1 & 10 \\
27 & 7 \\
02 & 10 \\
25 & 8 \\
12 & 10 \\
25 & 8\end{array}$} & \multirow{5}{*}{$\begin{array}{l}0.8975 \\
0.8975 \\
0.8975 \\
0.3975 \\
0.8930 \\
.5930 \\
0.8944 \\
0.8814 \\
0.8502 \\
0.8602\end{array}$} & & $0+18$ & & \\
\hline & & 0.8975 & $\left.\begin{array}{l}0.05 \\
0.15\end{array}\right\}$ & 0.42 & 0.5 \\
\hline & & & $\left.\begin{array}{l}0.05 \\
0.02\end{array}\right\}$ & 0.06 & 0 \\
\hline & & 0.3814 & 0.111 & 0.29 & 0.4 \\
\hline & & 0.8802 & $\begin{array}{l}0.06 \\
0.10\end{array}$ & 0.16 & .0 .4 \\
\hline \multirow{3}{*}{$\begin{array}{r}059 \\
344 \\
359 \\
372\end{array}$} & \multirow{3}{*}{ 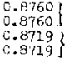 } & 0.5750 & 0.001 & 18 & \\
\hline & & 0.971 .8 & $0.25\}$ & 0.57 & 0.5 \\
\hline & & & 0.12 & & \\
\hline
\end{tabular}

Zur Überprüfung der aus Intensitätsrechnungen gewonnenen Ergebnisse wurde aus den experimentellen Daten der K-Verbindung zusätzlich eine dreidimensionale Fourier-Synthese berechnet (vgl. 1. c.11). Abbild. 1 zeigt die Elektronendichteverteilung in Schnitten parallel (001) durch die K-, O-, Si- und C-Atome. Aus Gründen der Übersichtlichkeit ist nur die Hälfte einer tetrameren Einheit dargestellt. Die Lagen der Elektronendichtemaxima entsprechen völlig den aus den Intensitätsrechnungen erhaltenen Werten.

\section{Beschreibung der Strukturen}

Die Trimethylsilanolate von $\mathrm{K}, \mathrm{Rb}$ und $\mathrm{Cs}$ sind isotyp und bilden wie die entsprechenden tert.-Butylate tetramere Aggregate. Dabei besetzen die Metall- und 
Tab. 3 (Fortsetzung)

\begin{tabular}{|c|c|c|c|c|}
\hline tis & $d_{\text {bex }}[\mathrm{i}]$ & 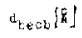 & $I_{\text {be }}$ & $I_{\text {beub }}$ \\
\hline $\begin{array}{ll}001 \\
01 & 1 \\
11 & 12 \\
\alpha & 2 \\
01 & 2 \\
11 & 2 \\
02 & 2 \\
0 & 3 \\
12 & 2 \\
01 & 3\end{array}$ & 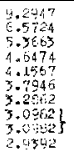 & $\begin{array}{l}9.25 \\
5.68 \\
5.850 \\
4.640 \\
4.156 \\
3.791 \\
3.255 \\
3.095 \\
2.997\end{array}$ & 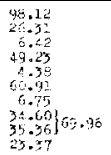 & $\begin{array}{c}100.0 \\
22 \\
4.9 \\
51 \\
3.3 \\
53 \\
6.0 \\
84 \\
26\end{array}$ \\
\hline $\begin{array}{ll}11 & 3 \\
22 & 2 \\
02 & 3 \\
12 & 3 \\
00 & 1 \\
22 & 3 \\
01 & 2 \\
03 & 3 \\
11 & 4 \\
15 & 3\end{array}$ & $\begin{array}{l}2.0025 \\
2.6032 \\
2.5779 \\
2.4841 \\
2.3257 \\
2.2543 \\
2.25<3 \\
2.3900 \\
2.1908 \\
2.1324\end{array}$ & $\begin{array}{l}2.201 \\
2.642 \\
2,5764 \\
2.4529 \\
2.3226 \\
2.2531 \\
2.1739 \\
2.1 \times 24\end{array}$ & 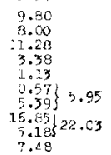 & $\begin{array}{r}9.13 \\
5.4 \\
21.2 \\
3.3 \\
3.5 \\
16.17 \\
22 \\
5.5\end{array}$ \\
\hline $\begin{array}{ll}02 & 4 \\
12 & 4 \\
23 & 3 \\
22 & 1 \\
00 & 5 \\
03 & 4 \\
01 & 5 \\
15 & 4 \\
11 & 5 \\
3 & 5\end{array}$ & $\left.\begin{array}{l}2.0784 \\
2.0243 \\
1.9616 \\
1.0973 \\
\left.\begin{array}{l}1.8509 \\
1.8549 \\
1.0228 \\
1.9220 \\
1.7828 \\
1.7408\end{array}\right\}\end{array}\right\}$ & $\begin{array}{l}3.0260 \\
1.9812 \\
1.8975 \\
1.0588 \\
1.8426 \\
1.7 \% 28\end{array}$ & 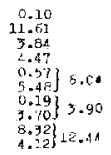 & $\begin{array}{l}0 \\
9.4 \\
2.5 \\
2.0 \\
6.4 \\
3.0 \\
11.7\end{array}$ \\
\hline $\begin{array}{ll}02 & 5 \\
23 & 4 \\
12 & 5 \\
04 & 4 \\
22 & 5 \\
14 & 4 \\
03 & 5 \\
33 & 4 \\
12 & 5\end{array}$ & 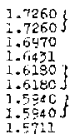 & $\begin{array}{l}1.7250 \\
1.5967 \\
1.6429 \\
1.51 \% 7 \\
=.5940 \\
1.5710\end{array}$ & $\left.\begin{array}{l}\left.\begin{array}{l}0.24 \\
1.69\end{array}\right\} 1.35 \\
6.30 \\
1.69 \\
2.01 \\
1.04 \\
0.05 \\
2.52\} \\
0.43 \\
0.43\end{array}\right\} 3.35$ & $\begin{array}{l}1.7 \\
6.4 \\
1.5 \\
3.5 \\
2.5 \\
0.7\end{array}$ \\
\hline 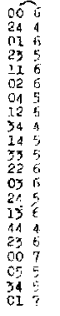 & 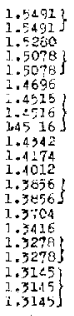 & $\begin{array}{l}1.1513 \\
1.4358 \\
1.3457 \\
1.3704 \\
1.3775 \\
1.3443\end{array}$ & 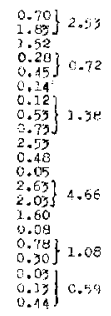 & $\begin{array}{l}3.2 \\
2.2 \\
0.9 \\
0 \\
1.2 \\
2.1 \\
0 \\
0 \\
4.4 \\
1.5 \\
0 \\
1.3 . \\
0.7\end{array}$ \\
\hline $\begin{array}{ll}15 & 5 \\
11 & 7 \\
04 & 5 \\
02 & 7 \\
14 & 0 \\
25 & 5 \\
5 & 5 \\
32 & 5 \\
30 & 6\end{array}$ & $\left.\begin{array}{l}1.3015 \\
1.5015\} \\
1.2869 \\
1.2767 \\
1.276 ?\} \\
1.2519 \\
1.2649 \\
1.2649 \\
1.2421\end{array}\right\}$ & $\begin{array}{l}1.7014 \\
1.2991 \\
1.2767 \\
2.2550\end{array}$ & 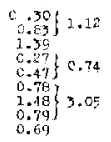 & $\begin{array}{l}1.6 \\
1.5 \\
0.7 \\
3.2 \\
0\end{array}$ \\
\hline 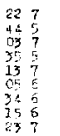 & $\left.\begin{array}{l}1.2311 \\
1.2312 \\
1.2085 \\
1.2101 \\
1.2101 \\
1.1901 \\
1.1501 \\
1.1804 \\
1.7504\end{array}\right\}$ & $\begin{array}{l}1.2509 \\
1.2204 \\
1.2209 \\
1.7903\end{array}$ & 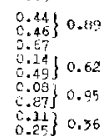 & $\begin{array}{l}0.8 \\
1.0 \\
0.6 \\
0.9 \\
0\end{array}$ \\
\hline $\begin{array}{ll}00 & 0 \\
25 & 6 \\
01 & 8 \\
04 & 7 \\
11 & 5 \\
11 & 6 \\
34 & 7 \\
33 & 7\end{array}$ & $\begin{array}{l}1.1613 \\
1.7529 \\
1.7529 \\
1.2529 \\
1.1419 \\
1.1441 \\
1.1441 \\
1.155\end{array}$ & $\begin{array}{l}1.1621 \\
1.1520\end{array}$ & $\left.\begin{array}{l}0.00 \\
0.09 \\
0.01 \\
0.19 \\
0.28 \\
0.51 \\
8.31 \\
8.56\end{array}\right\} 0.50$ & $\begin{array}{l}0.2 \\
1.3\end{array}$ \\
\hline
\end{tabular}

\begin{tabular}{|c|c|c|c|c|c|}
\hline hE & $\mathrm{d}_{\text {ber }}[2 \mathrm{j}$ & $a_{1, e o b}[R]$ & $I_{t e r}$ & & Iteob \\
\hline \multirow{13}{*}{ 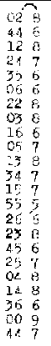 } & $\begin{array}{l}1.127 \times 3 \\
1.1272\end{array}$ & $x .12 ? \mathrm{~s}$ & 0.00 & 0.56 & 5.7 \\
\hline & $\begin{array}{l}1.1290 \\
1.1140\end{array}$ & 1.1 .143 & $0.58\}$ & 0.85 & 0.5 \\
\hline & $2.1: 09$ & & 0.16 & & $c$ \\
\hline & $\left.\begin{array}{l}2.0954 \\
\{.0954\end{array}\right\}$ & 1.0456 & $\left.\begin{array}{l}0.43 \\
0,80\end{array}\right\}$ & 0.72 & 0.9 \\
\hline & $\left.\begin{array}{l}1.0870 \\
1.0579\end{array}\right\}$ & $1.06 \mathrm{BI}$ & $\begin{array}{l}0.096 \\
0.16\}\end{array}$ & 0.39 & 0.5 \\
\hline & $\begin{array}{r}.0005 \\
0.0505\end{array} \mid$ & 1.0104 & $\begin{array}{l}0.02 \\
0.04\end{array}$ & 0.35 & $\therefore .6$ \\
\hline & $\begin{array}{l}.0865] \\
1.07331\end{array}$ & & 0.25 & & \\
\hline & $1.0133\}$ & 1.0134 & $\left.\begin{array}{c}0.13 \\
0.19\end{array}\right\}$ & 0.51 & 0.5 \\
\hline & 3.0562 & 1.0663 & 0.20 & & $\forall .54$ \\
\hline & $\left.\begin{array}{l}1.0542 \\
1.0502\end{array}\right\}$ & & $\begin{array}{c}c .03 \\
0.04\end{array}$ & 0.07 & 0 \\
\hline & 1.0525 & $\therefore . \mathrm{C}>25$ & 0.31 & & 6.4 \\
\hline & $\begin{array}{l}1.0542 \\
1.05<7 \text { ? }\end{array}$ & & 0.03 & & \\
\hline & $\left.\begin{array}{l}1.0527 \\
1.0327 \\
1.0527\end{array}\right\}$ & 1,0325 & $\begin{array}{l}0.38 \\
0.08 \\
0.12\end{array}$ & 0.93 & 1.0 \\
\hline 390 & $\left.\begin{array}{r}1.0264 \\
.0264\end{array}\right\}$ & $\therefore .0265$ & $\left.\begin{array}{l}c .32 \\
0,02\end{array}\right\}$ & 0.15 & 0.1 \\
\hline 357 & $\left.\begin{array}{l}\{.0202 \\
1.0202\end{array}\right\}$ & & $\left.\begin{array}{c}0.09 \\
0.09\end{array}\right\}$ & 0.12 & 0 \\
\hline 248 & 1.0141 & 7.0722 & 0.31 & & 0.3 \\
\hline $\begin{array}{ll}0 \\
05 & 7\end{array}$ & $\begin{array}{l}1.0002 \\
1.0012\end{array}$ & 1.0063 & 0.091. & 0.25 & 0.5 \\
\hline 556 & 1.0023 & & c.oj & & \\
\hline $\begin{array}{l}267 \\
129\end{array}$ & $\frac{1}{1.0003}$ & & 0.15 & 0.23 & $c$ \\
\hline 466 & 0.9908 & 0.3910 & 0.06 & & 0.6 \\
\hline $\begin{array}{ll}05 & 8 \\
22 & 9\end{array}$ & $\begin{array}{r}0.9822 \\
0.9852\end{array}$ & & 0.01 & & \\
\hline 267 & 0 & & 0.065 & 0.21 & 0 \\
\hline 049 & $\begin{array}{l}0.9852 \mathrm{~J} \\
0.979 \mathrm{a}\end{array}$ & & 0.021 & & \\
\hline & $\begin{array}{l}0.9796 \\
0.9798\end{array}$ & 0.9799 & 0.235 & 0.65 & 0.9 \\
\hline
\end{tabular}

Sauerstoffatome alternierend die Ecken eines nur geringfügig verzerrten Würfels. Dieser ist in Richtung der verlängerten Raumdiagonalen von den Trimethylsilylgruppen umgeben (vgl. Abbild. 2); dabei stehen die $\mathrm{CH}_{3}$-Gruppen ,auf Lücke“ zu den drei benachbarten Metallatomen.

Für die K- und Rb-Verbindung ergaben sich die Atomabstände und Valenzwinkel der Tab. 4.

Wie bei den anschließend beschriebenen massenspektrometrischen Untersuchungen gezeigt wird, liegen die Trimethylsilanolate von $\mathrm{K}, \mathrm{Rb}$ und $\mathrm{Cs}$ auch im Dampfzustand als Tetramere vor. 

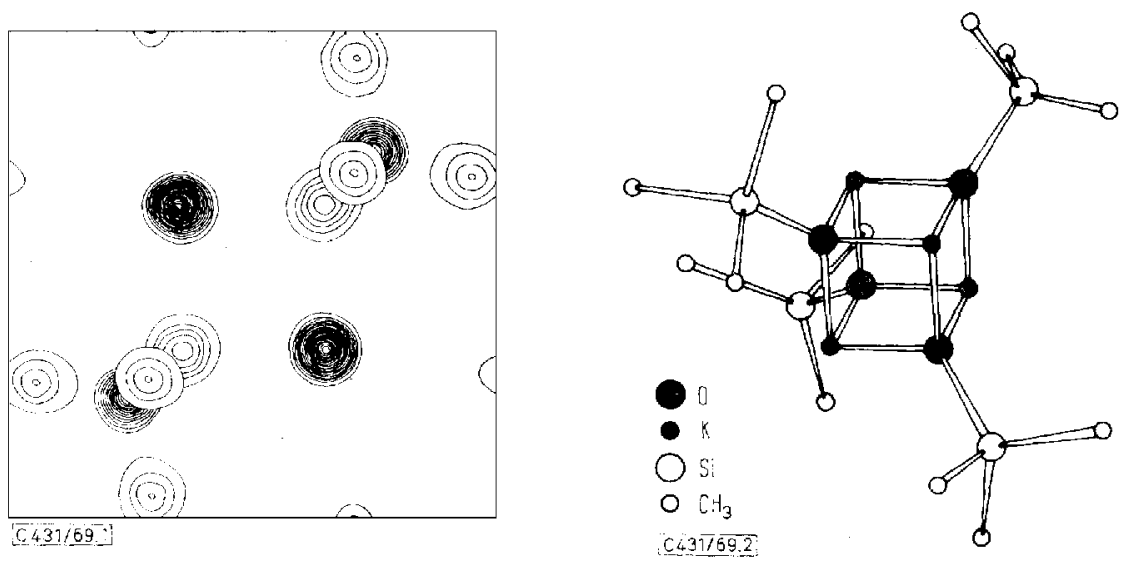

Abbild. 1. Fourier-Synthese von Kaliumtrimethylsilanolat (Projektion einer halben tetrameren Struktureinheit auf (001), kombiniert aus vier Schnitten durch die Atome)

Abbild. 2. Struktur des Kaliumtrimethylsilanolats (ohne H-Atome)

Tab. 4. Atomabstände und Valenzwinkel

\begin{tabular}{|c|c|c|}
\hline & {$\left[\left(\mathrm{CH}_{3}\right)_{3} \mathrm{SiOK}_{4}\right.$} & {$\left[\left(\mathrm{CH}_{3}\right)_{3} \mathrm{SiORb}_{4}\right.$} \\
\hline $\mathrm{M}-\mathrm{O}^{\mathrm{a})}$ & $2.61 \pm 0.04 \AA$ & $2.74 \doteq 0.06 \AA$ \\
\hline Si-Oa) & $1.60 \pm 0.06 \AA$ & $1.61^{b)}$ \\
\hline $\mathrm{Si}-\mathrm{CH}_{3}{ }^{\mathrm{a}}$ & $1.81=0.08 \AA$ & $1.83 \div 0.08 \AA$ \\
\hline \multirow[t]{2}{*}{$\left.\mathrm{CH}_{3}-\mathrm{CH}_{3} \mathrm{c}\right)$} & $3.87 \pm 0.08 \AA$ & $3.92 \_0.08 \AA$ \\
\hline & $4.29 \pm 0.08 \AA$ & $4.40 \pm 0.08 \AA$ \\
\hline $\mathrm{M}-\mathrm{O}-\mathrm{M}$ & $92 \pm 2^{\circ}$ & $93 \pm 3^{\circ}$ \\
\hline $\mathrm{O}-\mathrm{M}-\mathrm{O}$ & $\therefore 2^{\circ}$ & $\pm 3^{\circ}$ \\
\hline $\mathrm{M}-\mathrm{O}-\mathrm{Si}$ & $\pm 2^{\circ}$ & $\pm 3^{\circ}$ \\
\hline $\mathrm{O}-\mathrm{Si}-\mathrm{CH}_{3}$ & 1. $2^{\circ}$ & 1. $3^{\circ}$ \\
\hline $\mathrm{CH}_{3}-\mathrm{Si}-\mathrm{CH}_{3}$ & $\pm 2^{\circ}$ & $\pm 3^{\circ}$ \\
\hline
\end{tabular}

\section{Massenspektrometrische Untersuchungen *)}

Massenspektrometrisch konnten für das $\mathbf{L i}^{-6)}$ und das $\mathbf{K}$-tert.-Butylat ${ }^{1}$ in der Gasphase hexamere bzw. tetramere Einheiten nachgewiesen werden. Nach der gleichen Methode wurde jetzt der Assoziationsgrad der Alkalitrimethylsilanolate untersucht.

Die Proben wurden in einem offenen Goldtiegel in der Ionenquelle eines SM-1-Massenspektrometers (Varian MAT) so weit erhitzt, daß bei einer Elektronenenergie von $70 \mathrm{eV}$ hinreichend intensive Massenspektren erhalten wurden. Die Verdampfungstemperaturen betrugen für dic Li-Verbindung $50-60^{\circ}$, die Na-Verbindung $60-70^{\circ}$, die K-Verbindung $70-80^{\circ}$ und die Rb-Verbindung sowie Cs-Verbindung 80-100 $0^{\circ}$. Trotz dieser thermisch schonenden Behandlung konnte eine geringfügige Zersetzung zu Polysiloxanen nicht vermieden werden.

*) Durchgeführt von $H .-F$. Grützmacher.

6) G. E. Hartodt und T. L. Brown, lnorg. Chem. 5, 1257 (1966). 
Wegen der Isotopenzusammensetzung von $\mathrm{Si}$ sowie von $\mathrm{Li}, \mathrm{K}$ und $\mathrm{Rb}$ weisen die Massenspektren für jeden Ionentyp Peakgruppen auf. Für einen Vergleich der verschiedenen Alkalisilanolate müssen die Intensitäten der einzelnen Gruppen addiert werden. Der auf diese Weise berechnete Prozentanteil der verschiedenen Ionentypen am Gesamtionenstrom ist in Tab. 5 aufgeführt.

Tab. 5. Intensität der Ionentypen in den Massenspektren der Alkalitrimethylsilanolate $\left(\mathrm{R}==\left(\mathrm{CH}_{3}\right)_{3} \mathrm{SiO}\right)$, bezogen auf den Gesamtionenstrom.

\begin{tabular}{lccrrr}
\hline Ionentyp & LiR & NaR & KR & RbR & CsR \\
\hline $\mathrm{M}$ & \multicolumn{1}{c}{--} & - & 13.8 & 19.8 & 19.5 \\
$\mathrm{RM} \cdots \mathrm{CH}_{3}$ & 0.1 & 2.0 & 2.9 & 0.7 & 3.1 \\
$\mathrm{RM}$ & 3.1 & 31.4 & 40.0 & 36.8 & 41.2 \\
$\mathrm{R}_{2} \mathrm{M}_{2}-\mathrm{CH}_{3}$ & 11.9 & 36.4 & 37.3 & 32.2 & 27.8 \\
$\mathrm{R}_{2} \mathrm{M}_{3}$ & 0.2 & 1.0 & 0.3 & 1.0 & 1.3 \\
$\mathrm{R}_{3} \mathrm{M}_{3}-\mathrm{CH}_{3}$ & 0.4 & 0.8 & 0.3 & 1.4 & - \\
$\mathrm{R}_{3} \mathrm{M}_{4}$ & 5.2 & 4.2 & 5.0 & 7.7 & 7.1 \\
$\mathrm{R}_{4} \mathrm{M}_{4}-\mathrm{CH}_{3}$ & 34.7 & 21.6 & 0.4 & 1.3 & 0.1 \\
$\mathrm{R}_{4} \mathrm{M}_{5}$ & 0 & 0 & 0 & 0 & 0 \\
$\mathrm{R}_{5} \mathrm{M}_{5}-\mathrm{CH}_{3}$ & 1.3 & 0.4 & 0 & 0 & 0 \\
$\mathrm{R}_{5} \mathrm{M}_{6}$ & 0.1 & 0.3 & 0 & 0 & 0 \\
$\mathrm{R}_{6} \mathrm{M}_{6}-\mathrm{CH}_{3}$ & 43.2 & 1.9 & 0 & 0 & 0
\end{tabular}

Wie in den Massenspektren der Alkali-tert.-butylate ${ }^{1)}$ werden auch bei den Alkalitrimethylsilanolaten keine Ionen der Zusammensetzung $\mathrm{R}_{\mathrm{n}} \mathrm{M}_{\mathrm{n}}\left(\mathrm{R}=\left(\mathrm{CH}_{3}\right)_{3} \mathrm{SiO}\right.$, $\mathrm{M}=\mathrm{Li}, \mathrm{Na}, \mathrm{K}, \mathrm{Rb}$ oder $\mathrm{Cs}$ ) beobachtet, die den "Molekül-Ionen“ von Polymeren zugeordnet werden können. Wegen der leichten Abspaltung eines Silanolat-Anions treten nur $\left[R_{n-1} M_{n}\right]^{+}$-Ionen auf. Ein zweiter lonentyp $\left[R_{n} M_{n}-C H_{3}\right]^{+}$entsteht durch Abspaltung eines Methyl-Radikals aus den Molekül-Ionen. Diese Fragmentierung erfolgt bei den Trimethylsilanolaten deutlich häufiger als bei den entsprechenden tert.-Butylaten. Die leichte Abspaltung einer $\mathrm{CH}_{3}$-Gruppe des Trimethylsilylrestes ist aus den Massenspektren organischer Trimethylsilylderivate bekannt 7 .

Das Massenspektrum beweist für die $\mathrm{Li}$ - und die $\mathrm{Na}$-Verbindung das Vorliegen von Hexameren in der Gasphase. Die $\left[\mathrm{R}_{6} \mathrm{Li}_{6}-\mathrm{CH}_{3}\right]^{+}$-lonen liefern den stärksten Anteil am Gesamt-Ionenstrom. Daneben werden auch $\left[\mathrm{R}_{4} \mathrm{Li}_{4}-\mathrm{CH}_{3}\right]^{+}$- und $\left[\mathrm{R}_{2} \mathrm{Li}_{2}-\mathrm{CH}_{3}\right]^{+}-$ Ionen mit großen Intensitäten beobachtet. Es ist jedoch nicht bekannt, ob diese Ionen durch Elektronenstoß aus dem Hexameren entstehen, oder ob bereits beim Verdampfen eine thermische Dissoziation in Tetramere und Dimere und anschlieBende Ionisation erfolgt. Im Massenspektrum der Na-Verbindung sind $\left[\mathrm{R}_{6} \mathrm{Na}_{6} \cdots \mathrm{CH}_{3}\right]^{+}-$ Ionen ebenfalls deutlich, aber mit erheblich geringerer Intensität nachzuweisen als beim Li-Derivat. Dafür ist die Intensität der Ionen $\left[\mathrm{R}_{2} \mathrm{Na}_{2}-\mathrm{CH}_{3}\right]^{\div}$und besonders $\left[\mathrm{RNa}_{2}\right]^{+}$erhöht.

Ionen, die sich formal von einem Dimeren ableiten, geben auch in den Massenspektren der $K$-, $R b$ - und $C s$-Verbindung die größten Peaks. Dies stimmt mit dem Verhalten des K-tert.-Butylats überein. Die Ionen mit den höchsten m/e-Werten

7) A. G. Sharkey, R. A. Friedel und S. H. Langer, Analyt. Chem. 29, 770 (1957). 
entstehen bei diesen Silanolaten aus den Tetrameren, Hexamere lassen sich nicht nachweisen. Dieser Befund stimmt mit der röntgenographischen Untersuchung überein, bei der für die Trimethylsilanolate von $\mathrm{K}, \mathrm{Rb}$ und $\mathrm{Cs}$ ein aus tetrameren Einheiten aufgebautes Gitter gefunden wurde, während die entsprechende Li- und Na-Verbindung in einem anderen Gitter kristallisiert.

Bemerkenswert und möglicherweise charakteristisch für die Struktur der Alkalisilanolate im Gaszustand ist die Beobachtung, daß in den Massenspektren Ionen, die sich von $R_{n} M_{n}$ mit ungeraden $n$ ableiten, nur mit sehr geringer Intensität auftreten. Trimere und Pentamere sind offenbar unter diesen Bedingungen nicht stabil. Dies gilt auch für Lj-Trimethylsilanolat, bei dem eine Aufspaltung des Hexameren in zwei Trimere denkbar wäre. In den Massenspektren der beiden hexameren $\mathrm{Li}$ - und $\mathrm{Na}$-Trimethylsilanolate übertrifft die Intensität der Ionen $\left[\mathrm{R}_{\mathrm{n}} \mathrm{M}_{\mathrm{n}}-\mathrm{CH}_{3}\right]^{+}$stets die der Ionen $\left[\mathrm{R}_{\mathrm{n}-1} \mathrm{M}_{\mathrm{n}}\right]^{+}$, während bei den tetrameren Silanolaten leichter ein R-Anion als eine Methylgruppe abgegeben wird. Dieser Unterschied läßt sich besonders gut an den Ionen mit $n=4$ erkennen. Die Assoziate der Alkalitrimethylsilanolate werden demnach auch in der Gasphase durch die kleinen und stark polarisierenden Li- und Na-Ionen viel stärker zusammengehalten als durch die großen K-, Rb- und Cs-Ionen.

Wir danken dem Fonds der Chemischen Industrie für die Gewährung von Forschungsmitteln sowie der Hans-Heinrich-Hütte, Langelsheim/Harz, für die Überlassung von Alkalimetallen. 